\title{
Number of Subsets of the Set [n] Including No Three Consecutive Even Integers
}

\author{
Barış Arslan ${ }^{1 *}$, Kemal Uslu ${ }^{2}$ \\ 1* Selçuk University, Faculty of Science, Departmant of Mathematics, Konya, Turkey, (ORCID: 0000-0002-6972-3317), barismath@gmail.com \\ ${ }^{2}$ Selçuk University, Faculty of Science, Departmant of Mathematics, Konya, Turkey, (ORCID: 0000-0001-6265-3128), kuslu@selcuk.edu.tr
}

(1st International Conference on Applied Engineering and Natural Sciences ICAENS 2021, November 1-3, 2021)

(DOI: 10.31590/ejosat.1008742)

ATIF/REFERENCE: Arslan, B. \& Uslu, K. (2021). Number of Subsets of the Set [n] Including No Three Consecutive Even Integers. European Journal of Science and Technology, (28), 552-556.

\begin{abstract}
Consider an integer sequence counting the number of subsets of $S$ of the set $\{1,2, \ldots, n\}$ containing no three consecutive even integers. The sequence is associated with the Tribonacci sequence. Furthermore, we investigate some basic properties of the sequence.
\end{abstract}

Keywords: Tribonacci numbers, recurrence relation, consecutive even integers, generating function, combinatorial representation.

\section{[n] Kümesinin Ardışık Üç Çift Tam Sayı İçermeyen Alt Kümelerinin Sayısı}

Öz

$\{1,2, \ldots, n\}$ kümesinin ardışık üç çift tam sayı içermeyen $S$ alt kümelerinin sayısını veren tam sayı dizisini alalım. Bu dizi Tribonacci sayı dizisi ile ilişkilendirildi. Ayrıca dizinin bazı temel özellikleri incelendi.

Anahtar Kelimeler: Tribonacci sayıları, rekürans bağıntı, ardışık çift sayılar, üreteç fonksiyon, combinatoryal gösterim. 


\section{Introduction}

Fibonacci sequence and similar integer sequences are used in many fields from engineering to art. The Tribonacci numbers are a generalization of the Fibonacci numbers. Some properties of Tribonacci numbers are given in $[1,3,5,6,9,10]$.

The Tribonacci sequence $\left(T_{n}\right)_{n \geq 0}$ is defined by the thirdorder recurrence relation:

$$
\begin{aligned}
& T_{n}=T_{n-1}+T_{n-2}+T_{n-3}, \\
& T_{0}=0, T_{1}=1, T_{2}=1
\end{aligned}
$$

In [7] the Binet's formula for the Tribonacci sequence is given by

$T_{n}=\frac{\alpha^{n+1}}{(\alpha-\beta)(\alpha-\gamma)}+\frac{\beta^{n+1}}{(\beta-\alpha)(\beta-\gamma)}+\frac{\gamma^{n+1}}{(\gamma-\alpha))(\gamma-\beta)}$

where $\alpha, \beta$ and $\gamma$ are roots of the cubic equation $x^{3}-x^{2}-x-$ $1=0$, ie.,

$$
\begin{gathered}
\alpha=\frac{1+\sqrt[3]{19+3 \sqrt{33}}+\sqrt[3]{19-3 \sqrt{33}}}{3}, \\
\beta=\frac{1+\omega \sqrt[3]{19+3 \sqrt{33}}+\omega^{2} \sqrt[3]{19-3 \sqrt{33}}}{3}, \\
\gamma=\frac{1+\omega^{2} \sqrt[3]{19+3 \sqrt{33}}+\omega \sqrt[3]{19-3 \sqrt{33}}}{3},
\end{gathered}
$$

where $\omega=\frac{-1+i \sqrt{3}}{2}$ is a primitive cube root of unity.

"The number of subsets $\mathrm{S}$ of the set $[\mathrm{n}]=\{1,2, \ldots, n\}$ such that $\mathrm{S}$ contains no three consecutive integers." can be expressed in terms of the Tribonacci numbers. The answer is $T_{n+2}$ by obtaining a recurrence by considering those subsets $\mathrm{S}$ which do or do not contain the first element ' 1 '. By taking consecutive even integers instead of consecutive integers, we consider the following counting problem:

What is the number of subsets $S$ of the set [n]= $\{1,2, \ldots, n\}$ such that $\mathrm{S}$ contains no three consecutive even integers? In this paper, we denote the sequence by $\left(b_{n}\right)_{n \geq 0}$ corresponding to the counting problem.

After obtaining recursive definition of the sequence $\left(a_{n}\right)_{n \geq 0}$, we give the generating function, the closed form formula, the combinatorial representation and limit of the ratios of consecutive terms of the sequence.

\section{Main Results}

\subsection{Recursive definition of the sequence}

Let's write subsets $\mathrm{S}$ of the set $[\mathrm{n}]=\{1,2, \ldots, n\}$ such that $S$ contains no three consecutive even integers for some small $n$ values:
It's clear that for $n<6, b_{n}=2^{n}$. Since there are no three consecutive even integers for $n<6$. Hence, we get the initial conditions:

$$
b_{0}=1, b_{1}=2, b_{2}=4, b_{3}=8, b_{4}=16, b_{5}=32
$$

For $n=6$, there are $2^{6}-2^{3}=56$ subsets of the set $\{1,2,3$, $4,5,6\}$ such that $\mathrm{S}$ contains no three consecutive even integers. Hence, $b_{6}=56$.

Consider subsets counted by $b_{n}$. Let's find a recurrence for the sequence $\left(b_{n}\right)_{n \geq 0}$. For $n>5$ there are the following cases for the subsets:

1) The number of subsets not containing 2 as an element is $2 b_{n-2}$.

2) The number of subsets which contain 2, but don't contain 4 , is $4 b_{n-4}$.

3) The number of subsets which contain 2 and 4, but don't contain 6 , is $8 b_{n-6}$.

This gives a recurrence

$$
b_{n}=2 b_{n-2}+4 b_{n-4}+8 b_{n-6} \text {. }
$$

\subsection{Generating function and the Binet formula of the sequence}

Let the generating function associated to the sequence $\left(b_{n}\right)_{n \geq 0}$ be the formal power series

$$
F(x)=\sum_{n \geq 0} b_{n} x^{n}
$$

To find $F(x)$, multiply both sides of the recurrence relation (3) by $x^{n}$ and sum over the values of $n$ for which the recurrence is valid, namely, over $n \geq 6$. We get,

$$
\sum_{n \geq 6} b_{n} x^{n}=\sum_{n \geq 6} 2 b_{n-2} x^{n}+\sum_{n \geq 6} 4 b_{n-4} x^{n}+\sum_{n \geq 6} 8 b_{n-6} x^{n}
$$

Then try to relate these sums to the unknown generating function $F(x)$. We have,

$$
\begin{gathered}
\sum_{n \geq 6} b_{n} x^{n}=F(x)-b_{0}-b_{1} x-b_{2} x^{2}-b_{3} x^{3}-b_{4} x^{4}-b_{5} x^{5} \\
=F(x)-1-2 x-4 x^{2}-8 x^{3}-16 x^{4}-32 x^{5} \\
\quad \sum_{n \geq 6} 2 b_{n-2} x^{n}=2 x^{2} \sum_{n \geq 6} b_{n-2} x^{n-2} \\
=2 x^{2}\left(F(x)-b_{0}-b_{1} x-b_{2} x^{2}-b_{3} x^{3}\right) \\
=2 x^{2}\left(F(x)-1-2 x-4 x^{2}-8 x^{3}\right)
\end{gathered}
$$




$$
\begin{gathered}
\sum_{n \geq 6} 4 b_{n-4} x^{n}=4 x^{4} \sum_{n \geq 6} b_{n-4} x^{n-4}=4 x^{4}\left(F(x)-b_{0}-b_{1} x\right) \\
=4 x^{4}(F(x)-1-2 x) \\
\sum_{n \geq 6} 8 b_{n-6} x^{n}=8 x^{6} \sum_{n \geq 6} b_{n-6} x^{n-6}=8 x^{6} F(x)
\end{gathered}
$$

If we write these results on the two sides of (4), we find

$$
\begin{gathered}
F(x)-1-2 x-4 x^{2}-8 x^{3}-16 x^{4}-32 x^{5} \\
=2 x^{2}\left(F(x)-1-2 x-4 x^{2}-8 x^{3}\right)+4 x^{4}(F(x)-1-2 x) \\
+8 x^{6} F(x) .
\end{gathered}
$$

Which is trivial to solve for the unknown generating function $F(x)$ in the form

$$
F(x)=\frac{1+2 x+2 x^{2}+4 x^{3}+4 x^{4}+8 x^{5}}{1-2 x^{2}-4 x^{4}-8 x^{6}} .
$$

Theorem 1. For $n \in \mathbb{N}$, let $b_{n}$ be the number of subsets of $\mathrm{S}$ of the set $[n]=\{1,2, \ldots, n\}$ containing no three consecutive even integers. Then we have the following formulas for the subsequences of $\left(b_{n}\right)_{n \geq 0}$

$$
\begin{gathered}
b_{2 n}=2^{n} T_{n+2}, \\
b_{2 n+1}=2^{n+1} T_{n+2} .
\end{gathered}
$$

where $T_{n}$ is the $n$th Tribonacci number defined by (1).

Proof. If $A(x)$ is the generating function for even terms of the sequence $\left(b_{n}\right)_{n \geq 0}$, then it is clear that $A(x)=\frac{1}{2}(F(x)+F(-x))$. Substituting (5) we get,

$$
A(x)=\frac{1+2 x^{2}+4 x^{4}}{1-2 x^{2}-4 x^{4}-8 x^{6}}
$$

Substituting $u=2 x^{2}$ in (8) we have,

$$
A(u)=\frac{1+u+u^{2}}{1-u-u^{2}-u^{3}}
$$

The generation function of the Tribonacci sequence with initial conditions $T_{0}=1, T_{1}=1, T_{2}=2$ is

$$
\frac{1}{1-x-x^{2}-x^{3}} \text {. }
$$

$$
(1,1,2,4,7,13,24, \ldots) \leftrightarrow \frac{1}{1-x-x^{2}-x^{3}}
$$

Now let's right- shift the sequence (9) by adding 1and 2 leading zeros respectively:

$$
\begin{aligned}
& (0,1,1,2,4,7,13,24, \ldots) \quad \leftrightarrow \quad \frac{x}{1-x-x^{2}-x^{3}} \\
& (0,0,1,1,2,4,7,13,24, \ldots) \quad \leftrightarrow \quad \frac{x^{2}}{1-x-x^{2}-x^{3}}
\end{aligned}
$$

Let's try to obtain the generating function $A(x)$ using the generating functions of the Tribonacci sequences given in terms of initial conditions:

$$
\begin{gathered}
A(u)=\left(1+u+2 u^{2}+4 u^{3}+\ldots+T_{n+1} u^{n}+\ldots\right) \\
+\left(0+u+u^{2}+2 u^{3}+\ldots+T_{n} u^{n}+\ldots\right) \\
+\left(0+0 u+u^{2}+u^{3}+\ldots+T_{n-1} u^{n}+\ldots\right) \\
A(u)=\left(1+2 u+4 u^{2}+7 u^{3}+\ldots+T_{n+2} u^{n}+\ldots\right) \\
A(x)=\left(1+2\left(2 x^{2}\right)+4\left(2 x^{2}\right)^{2}+7\left(2 x^{2}\right)^{3}+\ldots\right. \\
\left.\quad+T_{n+2}\left(2 x^{2}\right)^{n}+\ldots\right) \\
A(x)=1+2.2 x^{2}+4.2^{2} x^{4}+7.2^{3} x^{6}+\ldots \\
\left.+T_{n+2} .2^{n} x^{2 n}+\ldots\right)
\end{gathered}
$$

Since $A(x)$ is the generating function for even terms of the sequence $\left(b_{n}\right)_{n \geq 0}$, we have

$$
b_{2 n}=2^{n} T_{n+2}
$$

where $T_{n}$ is the Tribonacci numbers with initial conditions;

$$
T_{0}=0, T_{1}=1, T_{2}=1 .
$$

If $B(x)$ is the generating function for odd terms of the sequence $\left(b_{n}\right)_{n \geq 0}$, then it is clear that $B(x)=\frac{1}{2}(F(x)-F(-x))$. Similarly using (5) and generating function method, for $n \geq 0$ we have

$$
b_{2 n+1}=2^{n+1} T_{n+2} .
$$

The proof is completed.

Corollary 1. For $n \in \mathbb{N}$, let $a_{n}$ be the number of subsets of $\mathrm{S}$ of the set $[\mathrm{n}]=\{1,2, \ldots, n\}$ including no three consecutive even integers. Then we have the following closed form formula

$$
b_{n}=2^{\left\lfloor\frac{n+1}{2}\right\rfloor} T_{\left[\frac{n+4}{2}\right\rfloor} .
$$

where $T_{n}$ is the $n$th Tribonacci number, $\lfloor n\rfloor$ is the floor of $n$.

Proof. Using Theorem 1, we can write piecewise defined sequence $\left(b_{n}\right)_{n \geq 0}$ asfollows:

$$
b_{n}= \begin{cases}2^{\frac{n}{2}} T_{\frac{n+4}{2}}, & \text { if } \mathrm{n} \text { is even } \\ 2^{\frac{n+1}{2}} T_{\frac{n+3}{2}}, & \text { if } \mathrm{n} \text { is odd }\end{cases}
$$

Then it is easy to see that

$$
b_{n}=2^{\left\lfloor\frac{n+1}{2}\right\rfloor} T_{\left[\frac{n+4}{2}\right\rfloor} .
$$

\subsection{Obtaining Binet formula of the sequence with combinatorial approach}

Let's try to find formulas respectively for the subsequences $\left(b_{2 n}\right)_{n \geq 0}$ and $\left(b_{2 n-1}\right)_{n \geq 1}$ of the sequence $\left(b_{n}\right)_{n \geq 0}$. Let's consider the set, $M=\{1,2,3, \ldots, 2 n\}$. For every $n \in \mathbb{N}$, let $a_{2 n}$ be the number of subsets of $\mathrm{S}$ of the set $M=\{1,2,3, \ldots, 2 n\}$ 
containing no three consecutive even integers. First, we separate the set $M$ into two disjoint subset $\mathrm{S}_{1}=\{1,3,5, \ldots, 2 \mathrm{n}-1\}$ and $S_{2}=\{2,4,6, \ldots, 2 n\}$. First notice that, counting subsets from $\mathrm{S}_{2}$ including no three consecutive even integers is equivalent to counting subsets from $\{1,2, \ldots, n\}$ including no three consecutive integers. Hence there are $T_{n+2}$ subsets where $T_{n}$ is the Tribonacci numbers defined by (1). The number of subsets of $S_{1}$ include no three consecutive even integers is equal to $2^{n}$ since all elements of $S_{1}$ are odd integers. Using multiplication principle, the total number of subsets of $M$ containing no three consecutive even integers is $2^{n} T_{n+2}$. Hence, we have

$$
b_{2 n}=2^{n} T_{n+2} \text {. }
$$

Considering the set, $M=\{1,2,3, \ldots, 2 n+1\}$ and using the same counting technique we have

$$
a_{2 n+1}=2^{n+1} T_{n+2} .
$$

\subsection{The combinatorial representation of the sequence}

The explicit formula of Tribonacci sequence is given in [4] by the formula

$$
T_{n}=\sum_{i=0}^{\left\lfloor\frac{n-1}{2}\right\rfloor} \sum_{j=0}^{i}\left(\begin{array}{l}
i \\
j
\end{array}\right)\left(\begin{array}{c}
n-1-i-j \\
i
\end{array}\right) .
$$

Using (6), (7) and (10) we have the combinatorial representation of the sequence $\left(b_{n}\right)_{n \geq 0}$

$$
\begin{array}{r}
b_{2 n}=2^{n} \sum_{i=0}^{\left\lfloor\frac{n+1}{2}\right\rfloor} \sum_{j=0}^{i}\left(\begin{array}{l}
i \\
j
\end{array}\right)\left(\begin{array}{c}
n+1-i-j \\
i
\end{array}\right), n \geq 0 \\
b_{2 n+1}=2^{n+1} \sum_{i=0}^{\left\lfloor\frac{n+1}{2}\right\rfloor} \sum_{j=0}^{i}\left(\begin{array}{l}
i \\
j
\end{array}\right)\left(\begin{array}{c}
n+1-i-j \\
i
\end{array}\right), n \geq 0 .
\end{array}
$$

Writing combinatorial identity for $2^{n}$ and using (11) and (12) we have,

$$
\begin{gathered}
b_{2 n}=\sum_{k=0}^{n}\left(\begin{array}{l}
n \\
k
\end{array}\right) \sum_{i=0}^{\left\lfloor\frac{n+1}{2}\right\rfloor} \sum_{j=0}^{i}\left(\begin{array}{l}
i \\
j
\end{array}\right)\left(\begin{array}{c}
n+1-i-j \\
i
\end{array}\right), n \geq 0 \\
b_{2 n+1}=\sum_{k=0}^{n+1}\left(\begin{array}{c}
n+1 \\
k
\end{array}\right) \sum_{i=0}^{\left[\frac{n+1}{2}\right]} \sum_{j=0}^{i}\left(\begin{array}{l}
i \\
j
\end{array}\right)\left(\begin{array}{c}
n+1-i-j \\
i
\end{array}\right), n \geq 0 .
\end{gathered}
$$

\subsection{Limit of the ratios of consecutive terms of the sequence}

It's well known that the limit of the ratio of two consecutive Fibonacci numbers is the Golden Ratio. A similar relationship occurs for the Tribonaccci numbers.

Define the sequence $\mathrm{x}_{\mathrm{n}}=\frac{T_{n+1}}{T_{n}}$ for $n \geq 1$ and $\lim _{n \rightarrow \infty} \mathrm{x}_{\mathrm{n}}=L$ exist. Using (1) for $n \geq 3$ we have

$$
\begin{gathered}
\mathrm{x}_{\mathrm{n}}=\frac{T_{n-2}+T_{n-1}+T_{n}}{T_{n}}=\frac{T_{n-2}}{T_{n}}+\frac{T_{n-1}}{T_{n}}+1, \\
\mathrm{x}_{\mathrm{n}}=\frac{T_{n-1}}{T_{n-1}} \frac{T_{n-2}}{T_{n}}+\frac{T_{n-1}}{T_{n}}+1, \\
\mathrm{x}_{\mathrm{n}}=\frac{1}{\frac{T_{n-1}}{T_{n-2}}} \frac{1}{\frac{T_{n}}{T_{n-1}}}+\frac{1}{\frac{T_{n}}{T_{n-1}}+1,} \\
\mathrm{x}_{\mathrm{n}}=\frac{1}{\mathrm{x}_{\mathrm{n}-2}} \frac{1}{\mathrm{x}_{\mathrm{n}-1}}+\frac{1}{\mathrm{x}_{\mathrm{n}-1}}+1 .
\end{gathered}
$$

Taking the limit of both sides of (1), we obtain $L=\frac{1}{L^{2}}+\frac{1}{L}+1$. Then $L^{3}-L^{2}-L-1=0$. We know that the terms of values of $T_{n}$ are real-valued and positive. From (13) we know that

$$
L=\frac{1+\sqrt[3]{19+3 \sqrt{33}}+\sqrt[3]{19-3 \sqrt{33}}}{3}
$$

is the only real-valued root of the equation $L^{3}-L^{2}-L-1=0$. Therefore,

$$
\begin{aligned}
\lim _{n \rightarrow \infty} \frac{T_{n+1}}{T_{n}}= & \frac{1+\sqrt[3]{19+3 \sqrt{33}}+\sqrt[3]{19-3 \sqrt{33}}}{3} \\
& \approx 1.839286755
\end{aligned}
$$

For any positive integer $\mathrm{k}$ and $\alpha=\frac{1+\sqrt[3]{19+3 \sqrt{33}}+\sqrt[3]{19-3 \sqrt{33}}}{3}$ the following limit is obtained in [1].

$$
\lim _{n \rightarrow \infty} \frac{T_{n+k}}{T_{n}}=\alpha^{k}
$$

Corollary 2. For $n \in \mathbb{N}$, let $b_{n}$ be the number of subsets of $\mathrm{S}$ of the set $[\mathrm{n}]=\{1,2, \ldots, n\}$ including no three consecutive even integers. Then we have the following results:

$$
\begin{gathered}
\lim _{n \rightarrow \infty} \frac{b_{2 n+1}}{b_{2 n}}=2 \\
\lim _{n \rightarrow \infty} \frac{b_{2 n}}{b_{2 n-1}}=\frac{1+\sqrt[3]{19+3 \sqrt{33}}+\sqrt[3]{19-3 \sqrt{33}}}{3},
\end{gathered}
$$

Proof. (16) is an immediate consequence of (6) and (7). (17) is implied by (6), (7) and (14).

Corollary 3. For $n \in \mathbb{N}$, let $b_{n}$ be the number of subsets of $\mathrm{S}$ of the set $[n]=\{1,2, \ldots, n\}$ including no three consecutive even integers. Then we have the following limit:

$$
\lim _{n \rightarrow \infty} \frac{b_{2 n+2 k}}{b_{2 n+1}}=2^{k-1} \alpha^{k}
$$

where $k$ is a positive integer and $\alpha=\frac{1+\sqrt[3]{19+3 \sqrt{33}}+\sqrt[3]{19-3 \sqrt{33}}}{3}$.

Proof. (18) is an immediate consequence of (6), (7) and (15). 


\section{Conclusions}

In this paper, we first obtained recursive formula of the sequence $\left(\boldsymbol{b}_{\boldsymbol{n}}\right)_{n \geq 0}$ which counts the number of subsets of $\mathrm{S}$ of the set $[\mathrm{n}]=\{\mathbf{1}, \mathbf{2}, \ldots, \mathbf{n}\}$ including no three consecutive even integers. Then we had the closed form formula of the sequence $\left(b_{n}\right)_{n \geq 0}$ using the generating function method and combinatorial approach. The combinatorial representation and limit of the ratio of consecutive terms of the sequence are obtained.

\section{References}

[1] Bueno, A. C. F. (2015). A note on generalized Tribonacci sequence, Notes on Number Theory and Discrete Mathematics, 21, 67-69.

[2] Feinberg, M. (1963). Fibonacci-Tribonacci, Fibonacci Quarterly, 1, 71-74.

[3] Pethe, S. (1988). Some Identities for Tribonacci sequences, Fibonacci Q., 26, 144-151.

[4] Ramirez, J. L. and Sirvent, V. F. (2014), Incomplete Tribonacci numbers and polynomials, Journal of Integer Sequences, 17 Article 14.4.2.

[5] Shannon, A. (1977). Tribonacci numbers and Pascal's pyramid, Fibonacci $Q ., 15,268-275$.

[6] Spickerman, W. and Joyner, R. N. Binets's formula for the Recursive sequence of Order K, Fibonacci Q., 22, 327-331.

[7] Spickerman, W. (1982). Binet's formula for the Tribonacci sequence, Fibonacci Q., 20, (118-120).

[8] Wilf, H. S. (1990). Generatingfunctionology, Academic Press.

[9] Yalavigi, C. C. (1972), Properties of Tribonacci numbers, Fibonacci Quarterly, 10 231-246.

[10]Yilmaz, N. and Taskara, N. (2014). Tribonacci and Tribonacci-Lucas Numbers via the Determinants of Special Matrices, Appl. Math. Sci., 8(39), 1947-1955. 